\title{
A SAÚDE DO TRANSGÊNERO E A CONTRIBUIÇÃO DA NUTRIÇÃO
}

\section{ESTADO DA ARTE}

COSTA, Melyssa de Souza e Silva ${ }^{1}$, CASIRAGHI, Bruna²

COSTA, Melyssa de Souza e Silva. CASIRAGHI, Bruna. A saúde do transgênero e a contribuição da nutrição. Revista Científica Multidisciplinar Núcleo do Conhecimento. Ano. 06, Ed. 12, Vol. 06, pp. 118-138. Dezembro de 2021. ISSN: 2448-0959, Link de acesso:

https://www.nucleodoconhecimento.com.br/saude/saude-do-transgenero, $\quad$ DOI: 10.32749/nucleodoconhecimento.com.br/saude/saude-do-transgenero

\section{RESUMO}

Para adequação de características que atendam ao gênero pertencente, ocorrem transformações corporais e psicossociais que implicam na saúde do transgênero. Diante disto, o presente artigo, tem como questão norteadora: como a nutrição pode estar envolvida na saúde do transgênero? O objetivo deste estudo é analisar o papel da nutrição sobre a saúde dos transgêneros, através do levantamento da literatura que aborde o assunto, para assim, vislumbrar caminhos e apontar as lacunas a serem preenchidas que permitam um atendimento mais abrangente e de qualidade a esta população, com ênfase na atuação do nutricionista. Como metodologia foi utilizada a revisão narrativa da literatura; utilizando-se as palavras-chave "transgênero e nutrição" e "transexual e nutrição" em busca nas bibliotecas virtuais científicas, sites e documentos envolvendo o tema e incorporado trabalhos em língua estrangeira. Como resultado foi notória a escassez de artigos que

\footnotetext{
${ }^{1}$ Pós-graduanda em Terapia Nutricional pela UFRJ, Pós-graduada em Nutrição Clínica, metabolismo, prática e terapia nutricional pela Faveni, Graduada em Nutrição pelo Centro Universitário de Volta Redonda- UNIFOA. ORCID: https://orcid.org/0000-0003-2845-8868.

${ }^{2}$ Orientadora. Doutora em Ciências da Educação, especialidade Psicologia da Educação, pela Universidade do Minho - Portugal, Mestre em Educação - Psicologia da Educação pela Pontifícia Universidade Católica de São Paulo, pós-graduada em Psicopedagogia pela UNIFOA e em Psicologia Jurídica pela UCAM, possui graduação em Faculdade de Psicologia PUC-SP pela Pontifícia Universidade Católica de São Paulo. Formada no Programa de Enriquecimento Instrumental níveis 1 e 2. ORCID: https://orcid.org/0000-0001-8114-3772.
}

RC: 103454

Disponível em: https://www.nucleodoconhecimento.com.br/saude/saude-dotransgenero 
abordassem a nutrição dos transgêneros diretamente na literatura brasileira. Entretanto, a nutrição pode estar envolvida ao processo de promoção da saúde dos transgêneros, visto que no processo de transição há implicações que o nutricionista pode intervir. Nesse sentido, os trabalhos apontam a importância do acolhimento e do atendimento integral aos transgêneros, assim como a necessidade de considerar as especificidades desta população no que diz respeito as estratégias e tratamentos que necessitam no processo de transição. Concluímos que a nutrição pode contribuir para promover saúde aos transgêneros, no entanto, há necessidade de mais estudos envolvendo a temática e o nutricionista necessita buscar conhecimento constante sobre esta população para melhor auxiliá-los.

Palavras-chave: Transgênero, Nutrição, Saúde.

\section{INTRODUÇÃO}

A identidade transgênero define-se pelo indivíduo que se distingue socialmente do gênero imposto ao nascer. Tal fato implica em uma segregação social, tendo em vista que, para uma sociedade pautada na binaridade dos gêneros, indivíduos que rompem essa dita normalidade são amplamente massacrados, acarretando assim problemas nas esferas biopsicossociais (SALLES; GONÇALVES; ARAÚJO, 2017).

Ao longo dos tempos, alguns direitos foram reivindicados pela população transgênero, tanto no campo social como na área da saúde, e alguns eventos históricos são relevantes para ilustrar esta busca de reconhecimento e identificação da transexualidade. A inclusão da transexualidade no Código Internacional de doenças (CID-10) no ano de 1980 configurou o início da sua patologização e, nesta mesma data, a transexualidade foi aprovada pela Associação Norte-americana como um "Transtorno de identidade de gênero". Contudo, em 2007, em Madri, Barcelona e Paris, iniciaram-se movimentos a favor da retirada da transexualidade como patologia, o que se estendeu por outros países, sendo que o Brasil passou a adotar essa campanha no ano de 2010 (BENTO; PELÚCIO, 2012). Em 29 de Janeiro de 2018, o Conselho Federal de Psicologia publicou uma resolução que ressaltava a 
necessidade do respeito as identidades de gênero, o combate à discriminação de transgêneros e a despatologização da transexualidade (CONSELHO FEDERAL DE PSICOLOGIA, 2019). Somente em 2019, durante a 72을 Assembleia Mundial de Saúde, que ocorreu em Genebra entre os dias 20 a 28 de maio, a transexualidade deixou, oficialmente, de ser classificada como doença ou transtorno mental, passando a ser classificada no CID-11 como "incongruência de gênero" (WHO, 2019).

No mundo, o preconceito e ideias pré-concebidas sobre transgênero e diversidade de gêneros acarretam uma opressão sobre esta parcela da população que contribuem para a privação de direitos básicos, violência de diversas formas e até assassinatos, estes não sendo precisamente estimados devido ao fato de que em diversos países não possuem uma sistematização na produção de dados relativo a pessoas Trans; ainda assim, em 2019 o Brasil liderou a posição de país que mais mata transgêneros, de acordo com o "Trans Murder Monitoring (TMM)", projeto que monitora, analisa e relata homicídios de pessoas transexuais e diversidade de gênero no mundo (BERREDO, 2019).

No âmbito da Saúde Pública no Brasil, devido às articulações e diálogos de organizações voltadas aos interesses da população Transexual e da diversidade sexual com Governo Federal, foram produzidas normas institucionais que contribuíram para o acesso e cuidado da população LGBTQI+, como a Política Nacional de Saúde Integral para População de Lésbicas, Gays, Bissexuais, Travestis e Transexuais, cujo objetivo é promover saúde Integral à essa população. No entanto, mesmo com avanços na área da saúde, ainda há desafios a serem combatidos como o subfinanciamento do SUS e setores conservadores que resistem a essas políticas (MONTEIRO; BRIGEIRO; BARBOSA, 2019).

Além das questões políticas e sociais, há uma trajetória individual que compreende a construção, aceitação do corpo e da personalidade na vida de pessoas Trans. $\mathrm{Na}$ construção de características que atendam ao gênero pertencente, podem ocorrer mudanças utilizando hormonioterapia, cirurgias, avaliações psicológicas, dentre 
outras intervenções que precisam da aproximação do usuário transgênero aos serviços de saúde; evidenciando que nesse processo de transição há aspectos biopsicossociais que necessitam ser acompanhados minuciosamente por uma equipe multiprofissional de saúde, no entanto, o estigma e a vulnerabilidade dessa população podem se configurar como fatores que dificultam o acesso a tais serviços (MONTEIRO; BRIGEIRO; BARBOSA, 2019).

Nesse contexto, o profissional da nutrição necessita estar atento às demandas na saúde desse grupo populacional para fornecer uma intervenção nutricional adequada. Para isso, a conscientização e ampliação do conhecimento podem promover cuidados nutricionais adequados à transgêneros, tendo em vista que os recursos para avaliações nutricionais, como as equações de quantidade energética, não são específicos para a população Trans, deixando exposto lacunas que necessitam ser preenchidas e evidenciando a necessidade de mais estudos que contemplem essa população (FERGUSSON et al., 2018). Nesta mesma linha, Rocon et al. (2020) aponta a necessidade de mais estudos científicos sobre o tema na área da saúde, destacadamente no que se refere à Política Nacional de Humanização do SUS. Este autor aponta, ainda, que os autores que abordam a temática do acesso da população Trans à saúde no Brasil entre 2008 e 2017 se repetem, o que pode configurar uma falta de diversidade de autores interessados na área. Diante do exposto, é nítida a necessidade de uma análise crítica da literatura sobre a transexualidade no que se refere ao acesso à saúde da população transgênero (ROCON et al., 2020) e abordagens que possam enfatizar especificidades no âmbito da Nutrição.

Desta forma, esta pesquisa se norteará pela seguinte questão: como a nutrição pode estar envolvida na saúde do transgênero? Portanto, o objetivo deste estudo é analisar o papel da nutrição sobre a saúde dos transgêneros, através do levantamento da literatura que aborde o assunto e análise crítica dos artigos, expressando o que os autores versam sobre a temática, possibilitando, assim, uma reflexão referente à saúde da população transgênero, com ênfase para a atuação do nutricionista.

RC: 103454

Disponível em: https://www.nucleodoconhecimento.com.br/saude/saude-dotransgenero 


\section{MÉTODOS}

Para esta pesquisa foi utilizada a Revisão Narrativa sobre estudos relacionados à saúde da população transgênero no campo da nutrição. Revisão Narrativa constitui um método científico amplo, que permite a discussão de um assunto sobre determinada óptica, constituindo-se na análise de informações contidas em artigos, livros, revistas e outras fontes de informações que apresentem confiabilidade para o seu desenvolvimento. As literaturas analisadas e interpretadas nesse tipo de revisão contribuem para construção de um ponto de vista, que pode ser pessoal do autor do estudo (ROTHER, 2007).

As referências analisadas neste estudo foram pesquisadas em artigos científicos de revistas eletrônicas, sites do Ministério da Saúde, periódicos, acesso em Biblioteca Virtual em Saúde no Brasil (BVS Brasil), LILACS, SciELO, PubMed, Arca Fiocruz e sites que envolvem a temática. Para a busca, utilizou-se os termos "Transgênero e Nutrição" e "Transexual e Nutrição" como palavras chaves para a pesquisa.

Como critérios de inclusão foram selecionados artigos que contemplassem 0 assunto transgêneros e nutrição. Foram excluídos artigos repetidos ou que não estavam em consonância com a temática proposta nesse estudo.

Após a seleção dos artigos e textos que contemplam a temática e sua interface com o campo da nutrição, os artigos foram lidos de forma a permitir uma análise crítica e os resultados foram apresentados nesse estudo, referenciando o autor da obra.

\section{RESULTADOS E DISCUSSÃO}

A busca na base de dados PubMed encontrou 71 artigos, no entanto apenas sete deles abordavam o tema na perspectiva da nutrição. Esses mesmos artigos também 
estavam disponíveis na BVS Brasil e no Portal de Periódicos CAPES. No LILACS foram encontrados 215 artigos com a palavra transgênero, porém nenhum artigo contemplava a nutrição relacionada a população pesquisada; o mesmo observado na biblioteca Virtual SciELO, assim como nas Revistas de Nutrição "Rasbran", Revista NUTR! Online e Revista Nature, onde também não foram encontrados nenhum artigo abordando as palavras chaves de busca.

Frente à escassez de trabalhos com o tema "transgênero e Nutrição" na literatura brasileira, ampliou-se a busca de referências com a inserção de palavra chave na língua Inglesa "transgender and Nutrition", não houve delimitação de data, contemplando artigos até o primeiro trimestre de 2020 e inseriu-se textos sobre "transexualidade e saúde" para estabelecer paralelos com o atendimento nutricional, os 8 artigos selecionados compõem o tópico "A garantia de acesso à saúde do Transgênero"; e selecionados 16 artigos que estabelecem relação direta com a proposta dessa pesquisa, e são discutidos no tópico "O processo de transição de gênero e a nutrição na promoção de saúde". Os artigos são descritos na tabela a seguir.

Figura 1. Tabela dos artigos selecionados

\begin{tabular}{|c|c|c|c|}
\hline Título & Autores & $\begin{array}{l}\text { Ano de } \\
\text { Publicação }\end{array}$ & Tipo de Artigo \\
\hline $\begin{array}{l}\text { Anna et al. Diet and Physical } \\
\text { Activity Behaviors Among }\end{array}$ & Bishop & 2020 & $\begin{array}{l}\text { Artigo de } \\
\text { Pesquisa }\end{array}$ \\
\hline \begin{tabular}{lcc} 
Adolescent & \multicolumn{2}{c}{ Transgender } \\
Students: & school & survey \\
results. : & School & Survey \\
Results & & \\
& &
\end{tabular} & & & \\
\hline $\begin{array}{l}\text { Understanding the Nutritional } \\
\text { Needs of Transgender and } \\
\text { Gender-Nonconforming }\end{array}$ & Kirby e Linde & 2020 & $\begin{array}{l}\text { Artigo } \\
\text { Pesquisa }\end{array}$ \\
\hline
\end{tabular}

RC: 103454

Disponível em: https://www.nucleodoconhecimento.com.br/saude/saude-dotransgenero 
Students at a Large Public

Midwestern University

(Re)escrevendo roteiros

(in)visíveis:

a

rajetória de

mulheres transgênero nas

políticas públicas de saúde

Chantal M et al. Fracture Risk

in Trans Women and Trans Men

Using Long-Term Gender-

Affirming Hormonal Treatment:

a nationwide cohort study: A

Nationwide Cohort Study

Título

Effects of sex steroids on cardiovascular risk profile in transgender men under gender affirming hormone therapy

Resolução № 2.265, DE 20 de Setembro de 2019

Barreiras de acesso na atenção primária à saúde à travestis e transexuais na região central de São Paulo

- Acesso das Pessoas Transexuais e Travestis à

\begin{tabular}{|l|l|l|}
\hline & $\begin{array}{l}\text { Antores } \\
\text { Publicação }\end{array}$ & Tipo de Artigo \\
\hline Aranda et al. & 2019 & $\begin{array}{l}\text { Artigo de } \\
\text { Pesquisa }\end{array}$ \\
\hline $\begin{array}{l}\text { Conselho } \\
\text { federal de } \\
\text { Medicina }\end{array}$ & 2019 & $\begin{array}{l}\text { Documento } \\
\text { Governamental }\end{array}$ \\
\hline $\begin{array}{l}\text { Magalhães } \\
\text { Pagsertação de }\end{array}$ \\
\hline $\begin{array}{l}\text { Pereira } \\
\text { Chazan }\end{array}$
\end{tabular}

Artigo de

Pesquisa

Artigo de

Pesquisa

\begin{tabular}{l|l|l} 
Wiepjes & 2020 & Artigo de \\
& & Pesquisa
\end{tabular}

Atenção Primária à Saúde: uma revisão integrativa

Nutrition-Related

Messages

Oliveira

e 2020

Romanini

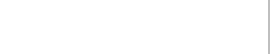




Shared Among the Online
Transgender Community: a
netnography of youtube
vloggers

Título
Impact of cross-sex hormone
therapy on bone mineral
density and body composition
in transwomen

Towards Providing Culturally Aware Nutritional Care for al Transgender People: key issues and considerations

Visibilidade trans na produção científica brasileira: contextos, temas, desafios e tendências

Corporalidades

Transgressoras: desafios das transformações corporais de pessoas transgênero no contexto da política social de saúde na Colômbia

Effect of Sex Steroids on the Bone Health of Transgender Individuals: a systematic review and meta-analysis

Título

\begin{tabular}{|l|l|l|}
\hline Autores & $\begin{array}{l}\text { Ano de } \\
\text { Publicação }\end{array}$ & Tipo de Artigo \\
\hline Figlera et al & 2018 & $\begin{array}{l}\text { Artigo d de } \\
\text { Revisão }\end{array}$ \\
& & \\
& & \\
\hline
\end{tabular}

Artigo de Rodrigues 2018

Revisão

Linsenmeyer

revisão

al.

Espitia-

2017

Pachón

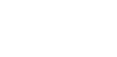

Singh-Ospina 2017

Artigo

de revisão

Autores
Ano de Tipo de Artigo
Publicação




\begin{tabular}{|c|c|c|c|}
\hline $\begin{array}{l}\text { A transexualidade na literatura } \\
\text { científica das ciências da } \\
\text { saúde }\end{array}$ & $\begin{array}{l}\text { Salles, } \\
\text { Gonçalves e } \\
\text { Araújo }\end{array}$ & 2017 & $\begin{array}{l}\text { Artigo de } \\
\text { Revisão }\end{array}$ \\
\hline $\begin{array}{l}\text { Corporal em } \text { Mulheres } \\
\text { Transgêneros Vivendo com } \\
\text { HIV/AIDS: uma discussão das } \\
\text { transformações que interferem } \\
\text { na avaliação do estado } \\
\text { nutricional }\end{array}$ & $\begin{array}{l}\text { Gonçalves et } \\
\text { al. }\end{array}$ & 2016 & $\begin{array}{l}\text { Artigo de } \\
\text { Revisão }\end{array}$ \\
\hline $\begin{array}{l}\text { Transexualidade } \\
\text { Travestilidade na Saúde }\end{array}$ & $\begin{array}{l}\text { Ministério da } \\
\text { saúde }\end{array}$ & 2015 & $\begin{array}{l}\text { Documento } \\
\text { Governamental }\end{array}$ \\
\hline $\begin{array}{l}\text { Guidelines and protocols For } \\
\text { Hormone Therapy and Primary } \\
\text { Health Care for Trans Clients }\end{array}$ & Bourns & 2015 & $\begin{array}{l}\text { Artigo de } \\
\text { Protocolo }\end{array}$ \\
\hline $\begin{array}{l}\text { Body composition, bone } \\
\text { turnover, and bone mass in } \\
\text { trans men during testosterone } \\
\text { treatment: 1-year follow-up } \\
\text { data from a prospective case- } \\
\text { controlled study (enigi) }\end{array}$ & $\begin{array}{l}\text { Van } \\
\text { Caenegem et } \\
\text { al. }\end{array}$ & 2015 & $\begin{array}{l}\text { Artigo de } \\
\text { Pesquisa }\end{array}$ \\
\hline Título & Autores & $\begin{array}{l}\text { Ano de } \\
\text { Publicação }\end{array}$ & Tipo de Artigo \\
\hline $\begin{array}{l}\text { Hormone therapy in } \\
\text { transgender adults is safe with } \\
\text { provider supervision; A review } \\
\text { of hormone therapy sequelae } \\
\text { for transgender individuals }\end{array}$ & $\begin{array}{l}\text { Weinand } \\
\text { Safer }\end{array}$ & 2015 & $\begin{array}{l}\text { Artigo } \\
\text { Revisão }\end{array}$ \\
\hline $\begin{array}{l}\text { Clinical management } \\
\text { transsexual subjects }\end{array}$ & $\begin{array}{l}\text { Costa } \\
\text { Mendonça }\end{array}$ & 2014 & $\begin{array}{l}\text { Artigo } \\
\text { Revisão }\end{array}$ \\
\hline Nutritional Status and Obesity & Vilas et al. & 2014 & Artigo \\
\hline
\end{tabular}




\begin{tabular}{|l|l|l|l|}
\hline $\begin{array}{l}\text { Prevalence in People with } \\
\text { Gender Dysphori }\end{array}$ & & & Pesquisa \\
\hline $\begin{array}{l}\text { Portaria no 2836/GM/MS, de 01 } \\
\text { de dezembro de 2011 }\end{array}$ & $\begin{array}{l}\text { Ministério da } 2011 \\
\text { saúde }\end{array}$ & $\begin{array}{l}\text { Documento } \\
\text { Governamental }\end{array}$ \\
\hline $\begin{array}{l}\text { Body composition, volumetric } \\
\text { and areal bone parameters in } \\
\text { male-to-female transsexual } \\
\text { persons }\end{array}$ & & 2008 & $\begin{array}{l}\text { Artigo de } \\
\text { Pesquisa }\end{array}$ \\
\hline
\end{tabular}

Fonte: Autores.

\section{A GARANTIA DE ACESSO À SAÚDE DO TRANSGÊNERO}

A abordagem científica utilizando o gênero como objeto de estudos iniciou-se na década de 70 em estudos feministas com o objetivo de buscar a igualdade de direitos entre homens e mulheres, questionando a posição ocupada pelas mulheres socialmente. Tais estudos contribuíram para uma reflexão sobre o binômio homem/mulher e, posteriormente, a outras questões transversais como violência, questões raciais e de classes, entre outros. Em relação à transgeneridade, ainda havia um regime normatizador baseado na binaridade; opondo-se a essa heteronormatização, surge a Teoria Queer, que amplia a questão do gênero, tornando-o mais plural, defendendo uma ideia de performance de gênero, da desconstrução de ideias sobre gênero e estimulando uma sociedade mais diversificada (SALLES; GONÇALVES; ARAÚJO, 2017).

A transexualidade era considerada uma patologia e obteve sua primeira conquista no campo da saúde no Brasil somente no ano de 1997, com a Resolução do Conselho Federal de Medicina que autorizava, ainda em caráter experimental, procedimentos para modificações de características secundárias e cirurgias de redesignação sexual para mulheres Trans, conhecida pela literatura como "Male to Female", sendo que a cirurgia de redesignação sexual para homens trans ("Female to Male") foi autorizada somente em 2010, ainda em caráter experimental. Os 
procedimentos hormonais e/ou cirúrgicos são autorizados mediante a necessidade de que as pessoas Trans preencham uma série de critérios. Tais normas estimulam a estigmatização da população trans e reforçam, também, um processo de exclusão dessas minorias, o que configura um contraponto ao que se propõe o SUS quando versa sobre universalidade e equidade (ROCON; SODRÉ; RODRIGUES, 2016).

Por meio da portaria ํㅡ 2.803, de 19 de novembro de 2013 (BRASIL, 2015), o processo transexualizador fica redefinido e ampliado, com atendimento psicológico e endocrinológico a transexuais de ambos os gênero e travestis, no entanto, por se tratar de uma portaria que pode ser revogada, pode gerar insegurança em relação ao direito adquirido.

$\mathrm{Na}$ busca de uma construção corporal que atenda ao seu gênero, alguns transgêneros acabam buscando procedimentos estéticos feitos por leigos, além da automedicação hormonal; nesse sentido, as políticas de saúde devem considerar a necessidade urgente de acesso integral de pessoas Trans pelo SUS, com objetivo de diminuir riscos associados aos procedimentos e contribuir para evitar a disseminação de opressões sociais na área da saúde (RODRIGUES, 2018). De encontro a isso, Pereira e Chazan (2019) expõem que o atendimento na saúde do transgênero deve ser ampliado para além do olhar sexualizador sobre o mesmo; visto que, constitui uma necessidade o atendimento multiprofissional a essa população, que deve ser iniciado na atenção básica, garantindo a facilidade de acesso e um acolhimento humanizado.

Salienta-se a relevância de estudos envolvendo esse tema, a fim de proporcionar uma visão mais ampla, para além de cirurgias e vulnerabilidade de doenças sexualmente transmissíveis, visto que o afunilamento da visão sobre essa população proporciona visões parciais e estereotipadas, ressaltando, assim, a saúde de forma integral, como preconiza o SUS (PEREIRA; CHAZAN, 2019). Nesse mesmo sentido, Oliveira e Romanini (2020) expressam a urgência de discussões que compreendam a população Trans na rotina diária dos serviços de saúde, com objetivo de evidenciar na prática o que a Política Nacional de Saúde LGBT já sugere; política 
essa que objetiva a promoção da saúde integral a população LGBT e está instituída no SUS, pela portaria GM/MS no 2.836/2011(BRASIL, 2011).

O conhecimento científico produzido na área da saúde é de grande relevância na vida de pessoas Trans, indo de encontro ao processo que a mesma passa na transição de gênero. No entanto, esse conteúdo científico sobre a transexualidade necessita caminhar em conjunto com as experiências que transgêneros vivenciam (OLIVEIRA; ROMANINI, 2020). Oliveira e Romanini (2020) reforçam a importância da educação constante dos profissionais de saúde, a fim de melhorar o acolhimento e 0 atendimento a pessoas Trans e consideram, ainda, necessário uma ampliação da interlocução entre o setor de especificidades em saúde com o setor de atenção primária, incluindo os agentes comunitários de saúde.

A recepção nas Unidades Básicas de Saúde caracteriza o primeiro contato do usuário transgênero com o SUS. Acolher o transgênero, não expondo-o a condições constrangedoras, contribui para sua inserção e continuidade no sistema. Um olhar menos cristalizado para o outro, desestimulando ideias pré-concebidas, valorizando o indivíduo e abrindo-se para a escuta sem certezas pré-estabelecidas descrevem a comunicação responsiva, que adotada na atenção primária ao usuário Transgênero tornam o ambiente menos discriminatório e transfóbico. Estimular e apoiar a continuidade dos serviços em saúde pode ser uma boa postura do profissional que deseja assegurar a integralidade à saúde do usuário transgênero, utilizando de estratégias pedagógicas para promover educação continuada a toda a equipe a fim de estreitar o contato do indivíduo transgênero no Sistema de saúde (MAGALHÃES, 2019).

\section{O PROCESSO DE TRANSIÇÃO DE GÊNERO E A NUTRIÇÃO NA PROMOÇÃO DE SAÚDE}

RC: 103454

Disponível em: https://www.nucleodoconhecimento.com.br/saude/saude-dotransgenero 
O Conselho Federal de Medicina, por meio da resolução ํㅡㄹ 2.265/2019, visa a dispor sobre o manejo específico na saúde do transgênero, contribuindo para o cuidado integral e multiprofissional; como cuidados relativos à saúde mental, procedimentos de cirurgias, caso necessário, e hormonioterapia, com o uso de antiandrógenos e estrógenos para mulheres Trans como feminilizantes e para Homens Trans o uso de testosterona como masculinizantes (BRASIL, 2019). A Terapia Hormonal constitui parte do processo de transição e, segundo Espitia-Pachon (2017), a construção de um corpo em pessoas Trans que atenda o gênero ao qual se identificam ocorrem mudanças desde a vestimenta até procedimentos mais invasivos; haja visto, que essa modificação corpórea é parte da promoção da saúde dessa população, pois contribuem para o bem-estar e equilíbrio dos aspectos biopsicossociais dos transgêneros.

Na Saúde, o suporte multidisciplinar que visa acompanhar e assegurar a utilização com cautela e segurança dessas medicações é imprescindível, visto que, muitas vezes, pela utilização de forma indiscriminada, tais condutas podem acarretar complicações de saúde. A utilização inadequada desses medicamentos pode acarretar excessos na ingestão, extrapolando a dosagem considerada adequada ou segura, na busca de resultados rápidos num curto intervalo de tempo, propiciando em uma overdose hormonal (ESPITIA-PACHÓN, 2017). Essa carga hormonal acima do recomendado, além de não ser necessária para atingir os objetivos, na maioria das vezes estão relacionados com efeitos secundários indesejados (COSTA E MENDONÇA, 2014).

$\mathrm{Na}$ busca da satisfação com a imagem, podem ocorrer adequações corporais que necessitam ser observadas, analisadas e inseridas no atendimento nutricional. Devido a hormonioterapia, é importante estar atento a aspectos como a composição corporal de mulheres transgênero, já que a Terapia Hormonal contribui para uma redistribuição corporal de massa magra e gorda. Outro fator relevante são as modificações corporais com o uso de géis de silicone e óleos de silicone que podem influenciar o Índice de Massa Corporal (IMC) e a Bioimpedância, podendo superestimar a massa gorda desses pacientes (GONÇALVES et al., 2016). 
Gonçalves et al. (2016) expressa, ainda, que os parâmetros utilizados para pessoas cisgêneros (indivíduos que expressa o gênero de acordo com o sexo biológico) não compreendem a pessoa transgênero, pois os parâmetros conhecidos como normalidade, são pautados na binaridade de gênero, portanto, essa dificuldade de se encaixar em parâmetros para população cis pode contribuir para que a avaliação antropométrica sugestione estados nutricionais equivocados.

A segurança da terapia hormonal é ainda assunto de discussão. Mesmo ainda necessitando de mais estudos e observações, Weinand e Safer (2015) concluem que a hormonioterapia é segura desde que respeitadas os cuidados e implicações adjacentes com uso dos hormônios, entretanto, o uso do estrógeno em mulheres trans apresentou risco associado a trombose, importância quanto a hipercolesterolemia, aumento nos níveis de triglicerídeos e baixa na libido. Já em homens trans, esses eventos não se relacionam com a terapia hormonal, mas em ambos os perfis apresentam uma elevação da glicemia em jejum e da resistência à insulina, além de uma modificação da gordura corporal (WEINAND; SAFER, 2015).

Bourns (2015) evidencia os riscos envolvendo a terapia hormonal, dentre eles os riscos de eventos cardiovasculares associados ao estrogênio e expressa que com o uso de hormônios exógenos a utilização do escore de Framingham pode apresentar menos confiabilidade. Aranda et al. (2019) identificou que, com o uso da testosterona, as mesmas observações são pertinentes e observou modificações nos indicadores metabólicos e de inflamação, que podem contribuir para complicações cardiovasculares em homens Trans. A identificação das complicações associadas e o tratamento farmacológico é importante para a segurança do tratamento hormonal; as doenças pré-existente, histórico familiar, dentre outros fatores, podem aumentar os riscos de câncer, sobretudo no que se refere a órgãos sexuais e/ou reprodutores da anatomia biológica de homens ou mulheres trans, além do risco para o câncer de mama, que pode se apresentar em ambos os casos (BOURNS 2015).

Os efeitos dos hormônios sobre a massa óssea do transgênero ainda é motivo de análise, no entanto, em mulheres trans houve aumento da Densidade Modular 
Óssea (DMO) na coluna lombar, não podendo afirmar que a diferença se relacione com possíveis fraturas, sendo que a terapia masculinizante não apresentou mudanças na densidade mineral óssea total do quadril (DMO) em homens Trans (SINGH-OSPINA et al., 2017). Também sobre este assunto, Van Caenegem et al. (2015) cita que os hormônios masculinizantes tiveram um efeito anabólico da massa muscular e óssea em homens trans.

No estudo de Lapauw et al. (2008) foi observado que com o uso da hormonioterapia, houve uma diminuição da massa magra e força muscular, enquanto ocorreu um aumento da massa gorda, o que indica que a saúde óssea de mulheres trans deve ser mais observada e acompanhada no tratamento a longo prazo, o que também é apontado por Wiepjes et al. (2020), que observaram, mesmo em mulheres trans mais novas, se comparadas com mulheres Cis, uma tendência ao risco de fraturas.

Bourns (2015) sugere no "Guidelines and Protocols for Comprehensive Primary Care for Trans Clients 2015" que em relação aos níveis de vitamina D e Cálcio é recomendado a garantia da ingestão, através da alimentação e suplementação, de 1000 Ul de Vitamina D e 1200mg de cálcio, e o estímulo de exercícios físicos podem ser eficazes na prevenção da osteoporose. No sentido de prevenir a perda óssea, pode ser benéfico em mulheres trans que fazem uso dos hormônios a longo prazo, as formas de terapias não medicamentosas aliados a um estilo de vida que favoreçam a manutenção da saúde óssea (FIGHERA et al., 2018).

O interesse de transgêneros em assuntos que envolvam dieta e exercícios físicos são amplamente difundidos nos meios eletrônicos, apoiando isso, Schier e Linsenmeye (2019), ao avaliar 30 vlogs públicos de pessoas trans entre 2013 e 2018, concluíram que os compartilhamentos de mensagens associadas à nutrição ocorrem amplamente, refletindo assim o interesse dessa população no conteúdo relacionado à nutrição. Porém, esse interesse pode não refletir o comportamento e hábitos alimentares de pessoas Trans; visto que, ainda que em uma amostra pequena, Kirby e Linde (2020) ao comparar estudantes transgêneros com os outros 
estudantes, identificaram que há uma prevalência negativa do consumo alimentar, de insegurança e de atitudes da autoimagem.

Em uma amostra de 157 indivíduos transgêneros em fase de tratamento hormonal, Vilas et al. (2014) identificaram um consumo energético alto na alimentação desses indivíduos, com consumo prevalente de gorduras saturada e colesterol e identificou aumento da gordura corporal em metade da população, sendo em maior frequência em mulheres trans; alguns participantes ainda relataram pular refeições como o café da manhã. $O$ estilo de vida e atitudes relacionadas a alimentação propicia um estado nutricional alterado, uma maior suscetibilidade a eventos cardiovasculares por conta do aumento do peso, ficando evidente que programas de educação nutricional precisam ser incluídos no tratamento multiprofissional que transgêneros recebem (VILAS et al., 2014).

Os programas de educação nutricional e esportiva necessitam desenvolver habilidades que comtemplem o público transgênero, pois atitudes negativas relacionadas à saúde foram identificadas em maior grau nos estudante trans do ensino médio quando comparados a estudantes cisgênero no estudo de Bishop et al. (2020); os autores destacam o consumo frequente de comidas e bebidas industrializadas e uma alimentação pobre em frutas e leite, além do sedentarismo muito presente e uma maior propensão ao sobrepeso e a obesidade.

Com relação a conduta nutricional adequada, Fergusson et al. (2018) evidenciam que o nutricionista deve reconhecer os riscos nutricionais e buscar continuamente desenvolver estratégias de aprimoramento na prática clínica para melhor atendimento, avaliação, tratamento e acompanhamento de pessoas trans; reconhecendo as probabilidade de apresentar risco nutricional elevado, além de intercorrências relacionadas à imagem corporal e inseguranças em relação à alimentação, que podem propiciar distúrbios alimentares e problemas relacionados a imagem, ainda nesse contexto, o profissional da nutrição necessitam ter uma postura com esses pacientes de empatia com a sua transição, estimulando uma vida saudável, desencorajando dietas rígidas e restritas, que podem desencadear 
possíveis transtornos alimentares. Mesmo que ainda haja na literatura escassez de estudo sobre a temática Trans, o estímulo de trabalhos de integração grupal pode gerar bons resultados. Como salientado por Fergusson et al. (2018), o nutricionista precisa estar consciente das questões que envolvem o transgênero para fornecer os devidos cuidados nutricionais.

\section{CONSIDERAÇÕES FINAIS}

O processo de transição de gênero implica em diversas modificações, que contemplam desde a vestimenta até procedimentos mais invasivos, como cirurgias estéticas e/ou de resignação sexual, hormonioterapia, entre outros. No âmbito do SUS, transgêneros contam com o amparo de uma equipe profissional que busca promover qualidade de vida e saúde para pessoas trans, no entanto, ainda existem pessoas trans que fazem os procedimentos de forma clandestina e sem segurança, o que evidencia a necessidade de atenção às demandas dessa população. $O$ estigma e a discriminação ainda é algo latente nesta temática. Na área da saúde, é necessário profissionais qualificados, que busquem conhecimento e atualização constantes a fim de promover um acolhimento e atendimento de qualidade para as pessoas e, em especial, para a parcela da população mais excluída e vulnerável.

Portanto, a questão que norteou esse estudo é evidenciada pelos artigos que expressam a relação da nutrição com a saúde dos transgêneros, no tocante as implicações do processo de transição; para isso, o profissional de nutrição necessita buscar conhecimento sobre transgêneros e as intercorrências da transição, reconhecer as limitações e tratar as complicações conforme protocolos nutricionais pré-estabelecidos. A nutrição tem papel importante na promoção de saúde para pessoas trans, visto que no processo de transição ocorrem implicações que o nutricionista pode intervir e promover a melhoria da qualidade de vida.

Por fim, nesta pesquisa concluímos que devido a escassez de artigos envolvendo a temática da nutrição dos transgêneros, a necessidade de usar palavras chaves na língua estrangeira, a repetição de autores que se interessam pelo tema e a 
necessidade utilizar assuntos correlacionados para explanar melhor o tema deixa exposto a urgência de mais estudos científicos na área de nutrição envolvendo transgêneros e o desinteresse acadêmico de elaborar estudos que envolvam a temática a partir da realidade brasileira. Desta forma, é importante que sejam realizados estudos para conhecer a realidade e adaptar os instrumentos usados na atuação do profissional da nutrição ao uso com a população trans, considerando que como os mesmos foram criados a partir do binômio homem/mulher, é provável, desta forma, que sua aplicação em clientes/usuários trans necessite de padrões próprios.

\section{REFERÊNCIAS}

ARANDA, Glória et al. Effects of sex steroids on cardiovascular risk profile in transgender men under gender affirming hormone therapy. Endocrinología, Diabetes y Nutrición, [s.I.], v. 66, n. 6, p. 385-392, jun. 2019. Elsevier BV. http://dx.doi.org/10.1016/j.endinu.2018.11.004.

BENTO, Berenice; PELÚCIO, Larissa. Despatologização do gênero: a politização das identidades abjetas: a politização das identidades abjetas. Revista Estudos Feministas, Florianópolis, v. 20, n. 2, p. 569-581, ago. 2012. FapUNIFESP (SciELO). http://dx.doi.org/10.1590/s0104-026x2012000200017.

BISHOP, Anna et al. Diet and Physical Activity Behaviors Among Adolescent Transgender Students: school survey results. : School Survey Results. Journal Of Adolescent Health, [s.I.], v. 66, n. 4, p. 484-490, abr. 2020. Elsevier BV. http://dx.doi.org/10.1016/j.jadohealth.2019.10.026.

BOURNS, Dr. Amy. GUIDELINES AND P R O T O C O L S For Hormone Therapy and Primary Health Care for Trans Clients. 2015. Disponível em: http://sherbourne.on.ca/wp-content/uploads/2014/02/Guidelines-and-Protocolsfor-Comprehensive-Primary-Care-for-Trans-Clients-2015.pdf. Acesso em: 30 abr. 2020.

RC: 103454

Disponível em: https://www.nucleodoconhecimento.com.br/saude/saude-dotransgenero 
BRASIL. CONSELHO FEDERAL DE MEDICINA (CFM). RESOLUÇÃO № 2.265, DE 20 DE SETEMBRO DE 2019: Dispõe sobre o cuidado específico à pessoa com incongruência de gênero ou transgênero e revoga a Resolução CFM № 1.955/2010. 2019. Disponível em: http://www.in.gov.br/en/web/dou/-/resolucao-n-2.265-de-20-desetembro-de-2019-237203294. Acesso em: 29 abr. 2020.

BRASIL, Ministério da Saúde. PORTARIA № 2.803, DE 19 DE NOVEMBRO DE 2013: Redefine e amplia o Processo Transexualizador no Sistema Único de Saúde (SUS). 2013.

Disponível em: http://bvsms.saude.gov.br/bvs/saudelegis/gm/2013/prt2803_19_11_2013.html. Acesso em: 25 abr. 2020.

BRASIL. MINISTÉRIO DA SAÚDE. Portaria no 2836/GM/MS, de 01 de dezembro de 2011.2011 .2 Disponível em: http://bvsms.saude.gov.br/bvs/saudelegis/gm/2017/MatrizesConsolidacao/comu m/13608.html. Acesso em: 29 abr. 2020.

BRASIL. MINISTÉRIO DA SAÚDE. Transexualidade e Travestilidade na Saúde. 2015.

Disponível em: http://bvsms.saude.gov.br/bvs/publicacoes/transexualidade_travestilidade_saud e.pdf. Acesso em: 29 abr. 2020.

CONSELHO FEDERAL DE PSICOLOGIA. Transexualidade não é transtorno mental, oficializa OMS: Decisão da OMS reforça a Resolução CFP no 01/2018, que orienta a atuação profissional de psicólogas(os) para que travestilidades e transexualidades não sejam consideradas patologias. Disponível em: https://despatologizacao.cfp.org.br/transexualidade-nao-e-transtorno-mentaloficializa-oms/. Acesso em: 16 abr. 2020.

COSTA, Elaine Maria Frade; MENDONCA, Berenice Bilharinho. Clinical management of transsexual subjects. Arquivos Brasileiros de Endocrinologia \& Metabologia, [s.I.], v. 58, n. 2, p. 188-196, mar. 2014. FapUNIFESP (SciELO). http://dx.doi.org/10.1590/0004-2730000003091.

RC: 103454

Disponível em: https://www.nucleodoconhecimento.com.br/saude/saude-dotransgenero 
ESPITIA-PACHÓN, Natalia. CORPORALIDADES TRANSGRESSORAS: desafios das transformações corporais de pessoas transgênero no contexto da política social de saúde na Colômbia, 2017, 155 f. Dissertação (Mestrado) - Curso de Pósgraduação em Políticas Públicas, Universidade Federal do Maranhão, São Luís, 2017

FERGUSSON, Pamela et al. Towards Providing Culturally Aware Nutritional Care for Transgender People: key issues and considerations. Canadian Journal Of Dietetic Practice And Research, [s.I.], v. 79, n. 2, p. 74-79, 1 jun. 2018.

FIGHERA, Tayane Muniz et al. Impact of cross-sex hormone therapy on bone mineral density and body composition in transwomen. Clinical Endocrinology, [s.l.], v. 88, n. 6, p. 856-862, 24 abr. 2018. Wiley. http://dx.doi.org/10.1111/cen.13607.

GONÇALVES, Juliana Lauar et al. COMPOSIÇÃO CORPORAL EM MULHERES TRANSGÊNERO VIVENDO COM HIV/AIDS: uma discussão das transformações que interferem na avaliação do estado nutricional. Demetra: Alimentação, Nutrição \& Saúde, [s.I.], v. 11, n., p. 1213-1223, 17 nov. 2016. Universidade de Estado do Rio de Janeiro. http://dx.doi.org/10.12957/demetra.2016.22542.

IBGE. Normas de Apresentação Tabular. 3. ed. Rio de Janeiro: Centro de Documentação e Disseminação da Informação, 1993. 62 p.

KIRBY, Sierra R.; LINDE, Jennifer A. Understanding the Nutritional Needs of Transgender and Gender-Nonconforming Students at a Large Public Midwestern University. Transgender Health, [s.I.], v. 5, n. 1, p. 33-41, 1 mar. 2020.

LAPAUW, Bruno et al. Body composition, volumetric and areal bone parameters in male-to-female transsexual persons. Bone, [s.I.], v. 43, n. 6, p. 1016-1021, dez. 2008. Elsevier BV. http://dx.doi.org/10.1016/j.bone.2008.09.001.

LUKAS BERREDO. Transrespect Versus Transphobia Worldwide (tvt) (comp.). Atualização TMM Trans Day of Remembrance 2019: 331 reported 
murders of trans and gender-diverse people in the last year. 2019. Disponível em: https://transrespect.org/en/tmm-update-trans-day-of-remembrance-2019/. Acesso em: 08 mar. 2020.

MAGALHÃES, Luiza Gomes. Barreiras de acesso na atenção primária à saúde à travestis e transexuais na região central de São Paulo. Universidade de São Paulo, São Paulo, p. 1-134, 2018. Universidade de Sao Paulo, Agência USP de Gestão da Informacao Academica (AGUIA). http://dx.doi.org/10.11606/d.7.2019.tde-08052019134851.

MEDICINA, Conselho Federal de. RESOLUÇÃO № 2.265, DE 20 DE SETEMBRO DE 2019: dispõe sobre o cuidado específico à pessoa com incongruência de gênero ou transgênero e revoga a resolução CFM № 1.955/2010. Dispõe sobre o cuidado específico à pessoa com incongruência de gênero ou transgênero e revoga a $\begin{array}{lllll}\text { Resolução CFM } & \text { no } & 1.955 / 2010 . & 2019 . & \text { Disponível }\end{array}$ em: http://www.in.gov.br/en/web/dou/-/resolucao-n-2.265-de-20-de-setembro-de2019-237203294. Acesso em: 29 abr. 2020.

MONTEIRO, Simone; BRIGEIRO, Mauro; BARBOSA, Regina Maria. Saúde e direitos da população trans. Cadernos de Saúde Pública, [s.I.], v. 35, n. 4, p. 1-4, abr. 2019. FapUNIFESP (SciELO). http://dx.doi.org/10.1590/0102-311x00047119.

OLIVEIRA, Itauane de; ROMANINI, Moises. (Re)escrevendo roteiros (in)visíveis: a trajetória de mulheres transgênero nas políticas públicas de saúde. Saúde e Sociedade, Rio Grande do Sul, v. 29, n. 1, p. 1-16, 2020. FapUNIFESP (SciELO). http://dx.doi.org/10.1590/s0104-12902020170961.

PEREIRA, Lourenço Barros de Carvalho; CHAZAN, Ana Cláudia Santos. O Acesso das Pessoas Transexuais e Travestis à Atenção Primária à Saúde: uma revisão integrativa. Revista Brasileira de Medicina de Família e Comunidade, Rio de Janeiro, v. 14, n. 41, p. 1-16, 14 maio de 2019. Sociedade Brasileira de Medicina de Familia e Comunidade (SBMFC). http://dx.doi.org/10.5712/rbmfc14(41)1795. 
ROCON, Pablo Cardozo et al. ACESSO À SAÚDE PELA POPULAÇÃO TRANS NO BRASIL: nas entrelinhas da revisão integrativa. : NAS ENTRELINHAS DA REVISÃO INTEGRATIVA. Trabalho, Educação e Saúde, [s.l.], v. 18, n. 1, p. 1-18, 2020. FapUNIFESP (SciELO). http://dx.doi.org/10.1590/1981-7746-sol00234.

ROCON, Pablo Cardozo; SODRÉ, Francis; RODRIGUES, Alexsandro. Regulamentação da vida no processo transexualizador brasileiro: uma análise sobre a política pública. : uma análise sobre a política pública. Revista Katálysis, [s.l.], v. $19, \quad$ n. $2, \quad$ p. 260-269, set. 2016. FapUNIFESP (SciELO). http://dx.doi.org/10.1590/1414-49802016.00200011.

RODRIGUES, Érica Gomes. Visibilidade trans na produção científica brasileira: contextos, temas, desafios e tendências. 2018. 109 f. Dissertação (Mestrado) Curso de Pós-graduação em Informação e Comunicação em Saúde, Instituto de Comunicação e Informação Científica e Tecnológica em Saúde, Rio de Janeiro, 2018.

ROTHER, Edna Terezinha. Revisão sistemática X revisão narrativa. Acta Paulista de Enfermagem, [s.I.], v. 20, n. 2, p. 1-2, jun. 2007. FapUNIFESP (SciELO). http://dx.doi.org/10.1590/s0103-21002007000200001.

SALLES, Débora Gomes; GONÇALVES, Jéssica dos Santos; ARAUJO, Luciana Danielli de. A transexualidade na literatura científica das ciências da saúde. Informação \& Informação, [s.I.], v. 22, n. 2, p. 265-292, 29 out. 2017. Universidade Estadual de Londrina. http://dx.doi.org/10.5433/19818920.2017v22n2p265.

SCHIER, Heather E.; LINSENMEYER, Whitney R. Nutrition-Related Messages Shared Among the Online Transgender Community: a netnography of youtube vloggers. Transgender Health, [s.I.], v. 4, n. 1, p. 340-349, 1 jan. 2019. Mary Ann Liebert Inc. http://dx.doi.org/10.1089/trgh.2019.0048. 
SINGH-OSPINA, Naykky et al. Effect of Sex Steroids on the Bone Health of Transgender Individuals: a systematic review and meta-analysis. The Journal Of Clinical Endocrinology \& Metabolism, [s.l.], v. 102, n. 11, p. 3904-3913, 13 set. 2017. The Endocrine Society. http://dx.doi.org/10.1210/jc.2017-01642.

VAN CAENEGEM, e et al. Body composition, bone turnover, and bone mass in trans men during testosterone treatment: 1-year follow-up data from a prospective casecontrolled study (enigi). European Journal Of Endocrinology, [s.I.], v. 172, n. 2, p. 163-171, fev. 2015. Bioscientifica. http://dx.doi.org/10.1530/eje-14-0586.

VILAS, María Victorina Aguilar et al. Nutritional Status and Obesity Prevalence in People with Gender Dysphoria. Aims Public Health, [s.I.], v. 1, n. 3, p. 137-146, 2014. American Institute of Mathematical Sciences (AIMS).

VOSGERAU, Dilmeire Sant'anna Ramos; ROMANOWSKI, Joana Paulin. Estudos de revisão: implicações conceituais e metodológicas. Revista Diálogo Educacional, [s.I.], v. 14, n. 41, p. 165-189, 12 jul. 2014. Pontificia Universidade Catolica do Parana - PUCPR. http://dx.doi.org/10.7213/dialogo.educ.14.041.ds08.

WEINAND, Jamie D.; SAFER, Joshua D. Hormone therapy in transgender adults is safe with provider supervision; A review of hormone therapy sequelae for transgender individuals. Journal Of Clinical \& Translational Endocrinology, [s.I.], v. 2, n. 2, p. 55-60, jun. 2015. Elsevier BV. http://dx.doi.org/10.1016/j.jcte.2015.02.003.

WIEPJES, Chantal $\mathrm{M}$ et al. Fracture Risk in Trans Women and Trans Men Using Long-Term Gender-Affirming Hormonal Treatment: a nationwide cohort study. Journal Of Bone And Mineral Research, [s.I.], v. 35, n. 1, p. 64-70, Jan. 2020. Wiley. http://dx.doi.org/10.1002/jbmr.3862.

WORLD HEALTH ORGANIZATION (WHO). Seventy-second World Health Assembly. 2019. Disponível em: https://www.who.int/classifications/icd/en/. Acesso em: 16 abr. 2020.

RC: 103454

Disponível em: https://www.nucleodoconhecimento.com.br/saude/saude-dotransgenero 
Enviado: Outubro, 2021.

Aprovado: Dezembro, 2021.

RC: 103454

Disponível em: https://www.nucleodoconhecimento.com.br/saude/saude-dotransgenero 